\title{
Cardiac innervation imaging: implications for risk stratification and therapeutic decision-making
}

\author{
M. Petretta ${ }^{1}$ (D)
}

Received: 19 June 2015/ Accepted: 25 June 2015/Published online: 7 July 2015

(C) Italian Association of Nuclear Medicine and Molecular Imaging 2015

Neurocardiology, the specialty that deals with the interaction between brain, heart, and autonomic nervous system, is a vivid area of research. It is well known that a lifethreatening stressor may induce a generalized autonomic storm, with both sympathetic and parasympathetic effects and the apparent predominance of one over the other depends on the variables considered, the timing of the observations and, probably, the type of the stressor. Moreover, remodeling of the cardiac nervous system influences the course of several cardiac diseases. As an example, cholinergic transdifferentiation of cardiac adrenergic neurons into cholinergic neurons, induced by leukemia inhibitory factor via a gp130 signaling pathway, supports the existence of potential plasticity of sympathetic neurons in heart failure (HF). The translational relevance of cardiac and extracardiac neural remodeling is under investigation and at this time many studies have demonstrated the value of autonomic indices to identify patients at risk for sudden death [1].

Relevant aspects of cardiac innervations, including preand postsynaptic receptors of the autonomic nervous system, are now investigated either by single-photon emission computed tomography (SPECT) and positron emission tomography (PET). In the setting of $\mathrm{HF}$, the prognostic relevance of cardiac neuronal imaging is under active evaluation. Several studies have indicated worse prognosis and higher risk of sudden death in patients with HF and reduced ${ }^{123}$ I-metaiodobenzylguanidine (MIBG) myocardial uptake or higher washout rate. Although dichotomizing the

M. Petretta

petretta@unina.it

1 Department of Translational Medical Sciences, Federico IIUniversity, Naples, Italy late $\mathrm{H} / \mathrm{M}$ ratio may be useful for stratifying patients into high-and low-risk categories, the patient survival rate decreases linearly accordingly to the impairment in cardiac MIBG activity. Moreover, the endpoint selection may influence the results observed in the setting of HF prognostication [2]. These challenges have slowed the wider clinical use of cardiac MIBG imaging, and the great potential of adrenergic system imaging still needs to be investigated in larger prospective studies before this technique can be implemented in clinical guidelines [3]. As an example, the importance of non-sudden cardiac death risk in predicting benefit from implantable cardioverter defibrillators (ICD) therapy must be considered. Due to the high cost of widespread ICD use, it is mandatory to identify a high-risk population who will benefit most from these devices. A number of non-left ventricular ejection fraction risk stratification tests for predicting a variety of outcomes (overall mortality, arrhythmic events/mortality, ICD shocks, and mortality benefit from ICD) are under evaluation. These tests include signal-averaged electrocardiogram, microvolt $\mathrm{T}$ wave alternans, electrophysiological testing, serum markers (including brain natriuretic peptide), and autonomic function evaluation (including heart rate variability, baroreflex sensitivity, heart rate turbulence, and deceleration capacity of heart rate). However, these tests have not demonstrated sufficiently high predictive value for arrhythmic death or arrhythmic events. Thus, it has been hypothesized that cardiac radionuclide imaging may be useful for identifying patients at risk for sudden cardiac death, therefore potentially offering a way to better select patients for ICD therapy. In patients with previous myocardial infarction, assessing the presence of an innervation/perfusion mismatch, i.e., a peri-infarction zone of sympathetic denervation that extends beyond the area of myocardial scar, as well as the presence of regional 
inhomogeneity of adrenergic innervations seems promising for identifying HF patients at risk for sudden cardiac death.

At this time, relatively little attention has been focused on the potential role of ${ }^{123}$ I-MIBG in guiding medical therapy of HF and avoidance of HF progression. With the growing burden of $\mathrm{HF}$, measuring the response of the cardiac sympathetic nervous system to treatment may help improve outcomes by optimizing HF medical therapy. However, many drugs may interfere with the uptake of MIBG. Also age-related reduction in $\mathrm{H} / \mathrm{M}$ ratios is to be considered while evaluating the results of imaging study. A further area of active investigation is in patients with advanced or end-stage HF. Interestingly, baseline late H/M ratio on ${ }^{123}$ I-MIBG planar imaging may predict cardiac resynchronization therapy response in these patients. It has been also suggested that ${ }^{123}$ I-MIBG imaging may be useful for predicting survival after transplant. However, if adrenergic imaging may be useful for prioritizing cardiac transplantation needs larger prospective studies before acceptance. Interestingly, the partial ventricular sympathetic reinnervation that may develop after transplantation can be measured using ${ }^{123} \mathrm{I}-\mathrm{MIBG}$ imaging as well by ${ }^{11} \mathrm{C}$ hydroxyephedrine $\left({ }^{11} \mathrm{C}-\mathrm{HED}\right) \mathrm{PET}$.

While cardiac MIBG imaging has a role in the risk assessment of patients with systolic left ventricular dysfunction, limited studies are available in patients with HF and preserved left ventricular ejection fraction and in those with pulmonary hypertension. Other potential applications of these techniques are in predicting development of chemotherapy-induced cardiomyopathy, myocardial recovery in patients with left ventricular assist devices, and assessing reinnervation following cardiac transplantation. Another potential field of research for cardiac innervation imaging is the study of right ventricular performance, in particular in the setting of pulmonary arterial hypertension. In fact, the role the right ventricle in the clinical presentation and course of several pathological states has been well recognized. However, at this time only a limited number of studies is available in patients with pulmonary hypertension.

The lack of standardization of ${ }^{123}$ I-MIBG imaging needs to be overcome for this imaging modality to gain popularity in clinical practice. The reproducibility of planar and of SPECT ${ }^{123}$ I-MIBG myocardial scintigraphy in patients with $\mathrm{HF}$ and left ventricular systolic dysfunction has been demonstrated. However, it must be outlined that in multicenter studies assessing the location of regions of interest may differ among institutions. Planar H/M ratio values based on medium-energy collimators are generally higher than that based on low-energy collimators. Thus, low-medium- energy collimators have been proposed to cover the higher energy scatter portion of the ${ }^{123}$ I energy spectrum, in order to achieve good balance among resolution, sensitivity, and applicable energy range. Using calibration phantom experiments and measured conversion coefficients specific for individual camera-collimator systems, all H/M ratios could be converted to standardized H/M ratios. Perhaps, new SPECT-only cameras, such as those based on solid-state Cadmium-Zinc-Telluride detectors, are now suitable to be used for MIBG imaging. These new scanners may improve the quantitative MIBG accuracy due to higher photon energy resolution, with better ability to discriminate scattered photons.

\section{Compliance with Ethical Standards}

Conflict of interest The author declares not to have conflict of interest.

Ethical standards This article does not contain any studies with human or animal subjects performed by any of the Authors.

\section{References}

1. Fukuda K, Kanazawa H, Aizawa Y, Ardell JL, Shivkumar K (2015) Cardiac innervations and sudden cardiac death. Circ Res 116:2005-2019

2. Petretta M, Pellegrino T, Cuocolo A (2014) Cardiac neuronal imaging with ${ }^{123}$ I-meta-iodobenzylguanidine in heart failure: implications of endpoint selection and quantitative analysis on clinical decisions. Eur J Nucl Med Mol Imaging 41:16631665

3. Saba S, Aban I, Soman P (2014) ${ }^{123}$ I-mIBG scintigraphy: yet another risk stratifier for the heart failure toolbox! J Nucl Cardiol 21:909-912 